\title{
Research on Intelligent Vehicle Collision Warning Model Based on Intervehicle Communication
}

\author{
Jiangfeng Wang, Xuedong Yan, Shuo Nie, and Xiaomeng Li \\ MOE Key Laboratory for Transportation Complex Systems Theory and Technology, Beijing Jiaotong University, \\ 3 Shangyuancun, Xizhimenwai, Beijing 100044, China \\ Correspondence should be addressed to Jiangfeng Wang; wangjiangfeng@bjtu.edu.cn
}

Received 13 November 2012; Accepted 8 January 2013

Academic Editor: Valentina E. Balas

Copyright (c) 2013 Jiangfeng Wang et al. This is an open access article distributed under the Creative Commons Attribution License, which permits unrestricted use, distribution, and reproduction in any medium, provided the original work is properly cited.

\begin{abstract}
Using inter-vehicle communication, vehicle real-time traveling state information, such as acceleration, is shared, and time-tocollision and time-to-avoidance matrices are established. Considering driver type and traveling relationship between leading vehicle and following vehicle, a collision warning model based on inter-vehicle communication is studied. From aspects of inter-vehicle experiment system composition, component modules are analyzed in detail. For leading vehicle stationary, sudden deceleration, and normal traveling, three experimental schemes are proposed, and model warning performance is verified using the experiment system. Experiment results show that the algorithm model can effectively identify the collision, and precise warning rate is more than $90 \%$. Conclusion of the study can provide a reference for research in relevant fields, such as vehicle adaptive cruise control.
\end{abstract}

\section{Introduction}

Road traffic safety has become an important factor to threaten people's lives and property, and it attracts more and more attention of government departments and traffic researchers. From 2000 to 2010, there were a total of 5265618 car accidents in china, in which 4585451 people were injured and 989140 people were killed [1]. In order to reduce traffic accidents, researchers design intelligent vehicle collision warning systems (CWSs) to avoid accidents based on radar, video and communications technologies. CWSs based on intervehicle communication with non-line-of-sight (NLOS) avoidance capacity are more suitable for providing warning information for multilane conditions [2]. Using reliable information transmission mechanism, one can effectively improve the performance of CWSs.

In recent years, intervehicle communication technology is widely used in CWSs studies, and some novel warning model algorithms are also presented by researchers. Based on DSRC, Biswas and Antony designed collaborative CWSs using collision avoidance time (time to avoidance, TTA) to achieve effective vehicle warning, respectively $[3,4]$. Considering the driver experience, Inoue et al. proposed a warning model based on brake prediction [5]. Supposing two vehicles maintaining the current speed and direction, the vehicle collision time (time to collision, TTC) is predicted to issue effective warning [6]. Instead of TTC, Miller et al. studied a cooperative intersection collision warning mode using TTA that is not limited by the requirement of line-of-sight [7]. While common TTA and TTC matrix are established without the vehicle acceleration information in previous literatures, which results in the inaccurate and impractical vehicles travelling information and have a high false alarm rate. In addition, some researchers proposed several corresponding collision warning models using new method such as fuzzy neural network [8].

Considering vehicle speed, acceleration, and other information, this paper designs a novel collision warning model based on the communication; intervehicle communication experimental system is provided, and the key modules of experimental system are analyzed. Using the experiment system, the model is validated under real field conditions combined with the proposed experimental scheme.

\section{Warning Model Analysis}

2.1. Theory Analysis. A collision accident is caused by driver's inattention to a great extent, which results in insufficient 
response time in some emergency and makes it difficult to avoid vehicle collision in a limited time. CWSs are used to assist drivers to deal with unexpected traffic conditions such as emergency parking, to identify collision, and then to provide real-time information to avoid collision accident.

Warning system effectiveness depends mainly on realtime acquisition and interaction of vehicle driving state parameters, in which velocity and acceleration are important parameters for establishing critical vehicle warning model. The former methods that acquire distance parameter based on Doppler effect, then obtaining vehicle speed and acceleration information by differential, have a big data error and cannot meet CWSs low delay rate requirements because of its complex data processing course.

Using intervehicle communication, travelling state information between vehicles are real-time transmitted with a low delay characteristic [9]. Vehicles installing wireless communication module can build a vehicular ad hoc networks in a certain areas; CWSs based on communication can effectively realize the interaction of vehicle running state information, including vehicle position, velocity, acceleration, and other information. Based on the TTC and TTA matrices, a rational collision warning model is established, which recognizes the collision and provides the collision warning information for drivers. The collision warning model logic frame based on intervehicle communication is shown in Figure 1.

In the process of vehicles' driving, it compares TTC with TTA in real time and determines whether to issue the warning information according to different driver types and

$$
\text { TTC }- \text { TTA }<\gamma
$$

where $\gamma$ is the release time adjusting parameter of warning information, with different value according to different driver type. Its value represents the concise extent of the warning information issued by the warning algorithm model, and the bigger means more conservative and more likely to cause interference.

2.2. Model Establishment. When the model is designed, TTC and TTA decision matrices are established using the acceleration information and so on, and decision is made based on the relationship between TTC and TTA matrices.

$v_{L}, v_{F}$ denote velocity of leading vehicle and following vehicle, respectively, and $a_{L}, a_{F}$ denote acceleration of leading vehicle and following vehicle, respectively. The running state information of leading vehicle and following vehicle is shared to each other by communication. In the traveling state, the relative velocity, acceleration, distance between leading vehicle and following vehicle at any time are denoted by $v_{r}, a_{r}$, and $d_{r}$, respectively. At the TTC, two vehicles may be collided at $\mathrm{S}$ point according to the current running state. The whole running process is shown in Figure 2.

Using traffic relation equation, collision time TTC at S point is obtained according to

$$
\mathrm{TTC}=\frac{-v_{r}+\sqrt{v_{r}^{2}+2 a_{r} d_{r}}}{a_{r}},
$$

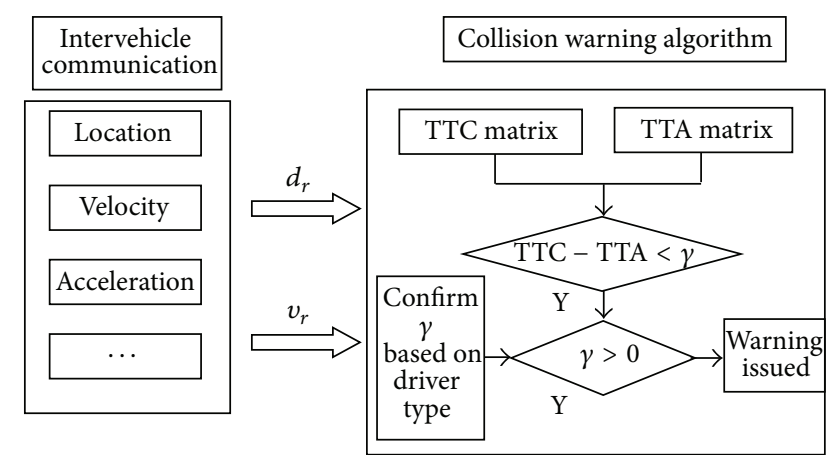

FIGURE 1: Collision warning logic diagram based on intervehicle communication.

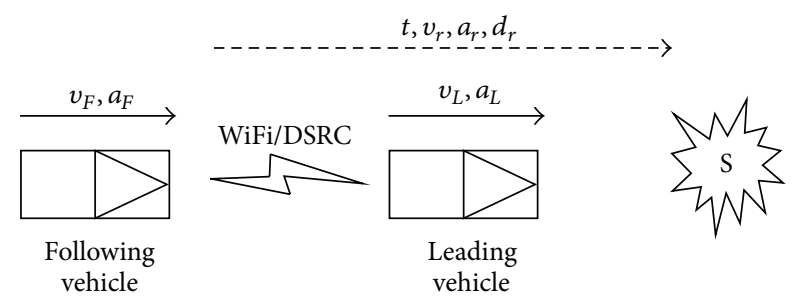

FIGURE 2: Vehicle traveling process.

where $d_{r}$ is the distance between two vehicles $(\mathrm{m}) ; v_{r}$ is the relative velocity of two vehicles $(\mathrm{m} / \mathrm{s}) ; a_{r}$ is the relative acceleration of two vehicles $\left(\mathrm{m} / \mathrm{s}^{2}\right)$.

TTA depends on many factors such as vehicle dynamics performance and driver reaction time. Selecting parameters of reaction time, braking performance and the safety headway, TTA is

$$
\mathrm{TTA}=t_{r}+\frac{\alpha v_{F}}{\mu g}+t_{h}
$$

where $t_{r}$ is driver reaction time (s), including driver's reaction time and brake reaction time. Due to individual difference, drivers have different judgment time generally, $0.56 \mathrm{~s}$ is supposed to be the safe reaction time. $\alpha$ is the velocity slow coefficient which denotes the relative distance after braking, and its range is $(0,1] ; \alpha=1$ indicates leading vehicle is in stop state; $g$ is the acceleration of gravity $\left(\mathrm{m} / \mathrm{s}^{2}\right) ; \mu$ is the adhesion coefficient which is related to road pavement types and status; this the safety vehicle headway (s) which has a little change range, and its value is $2 \mathrm{~s}$ [10].

After obtaining the TTC and TTA matrix, one determine whether to issue the warning information.

\section{Experimental System Design}

Choosing the JN5139 as a vehicular wireless communication master chip, the entire system mainly includes four key modules: power voltage regulator module, communication module, information display module, and the vehicle location module, as shown in Figure 3. 


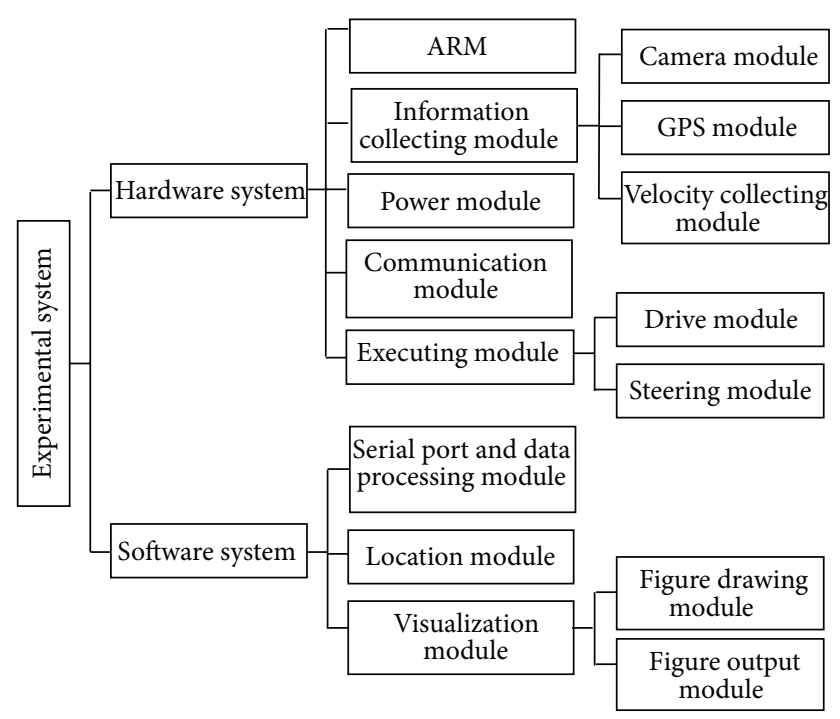

FIGURE 3: Intervehicle communication experiment system structure diagram.

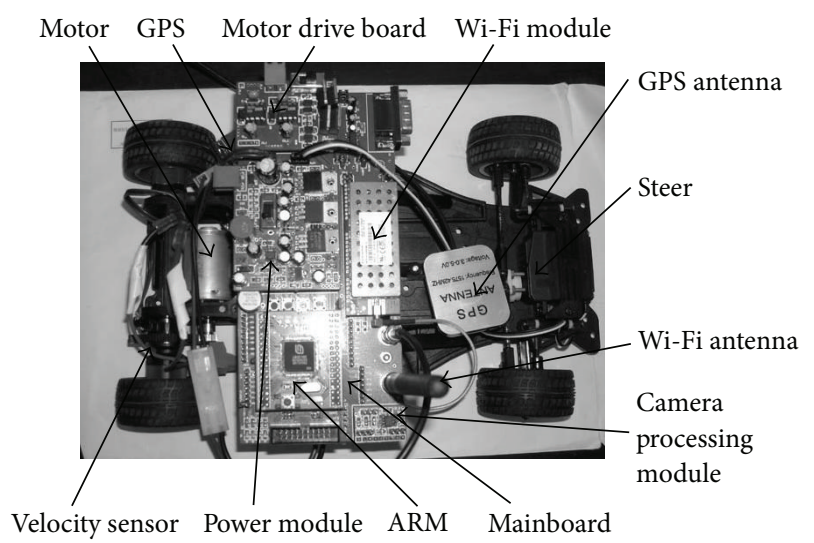

FIGURE 4: System hardware structure diagram.

3.1. Experimental Hardware System. The system hardware is mainly responsible for the transmission of information and collection, including a main control module, an information acquisition module, power supply module, wireless communication module, data processing module, the execution module, and GPS module, as shown in Figure 4.

3.2. Experimental Software System. The system is mainly responsible for information display, storage, and processingm and so forth, functions. Real-time data is sent to the supervisory computer by master chip through the serial port. By analyzing and calculating the receiving data, the supervisory computer displays the vehicle travelling information. Supervisory computer and display module program are compiled using VB 6.0, and program interface can dynamically display the location of vehicles, as shown in Figure 5.

3.3. Power Voltage Regulator Module. Experiment system is used in vehicles, and operating voltage of the whole system

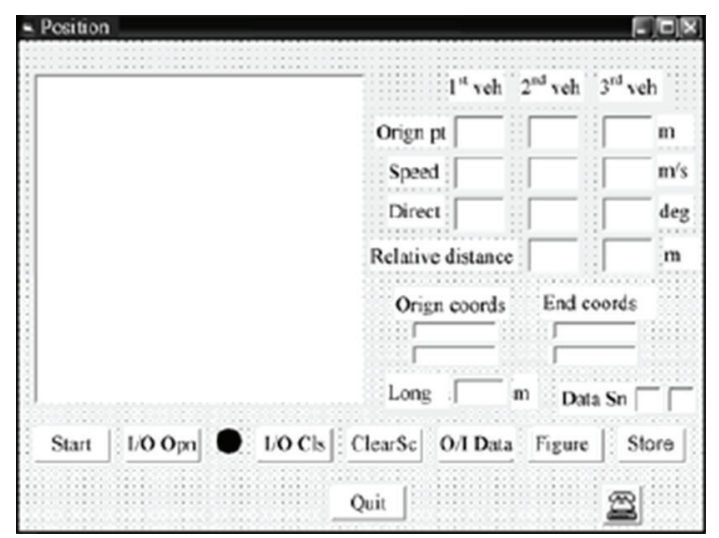

FIGURE 5: Display module interface.
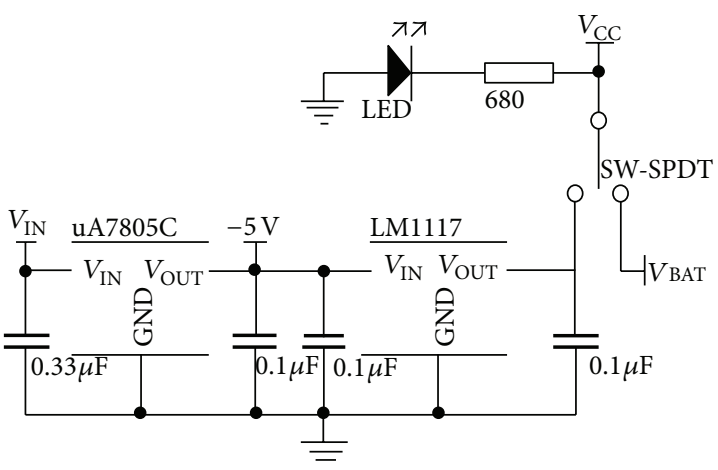

FIGURE 6: Power voltage stabilizing circuit module.

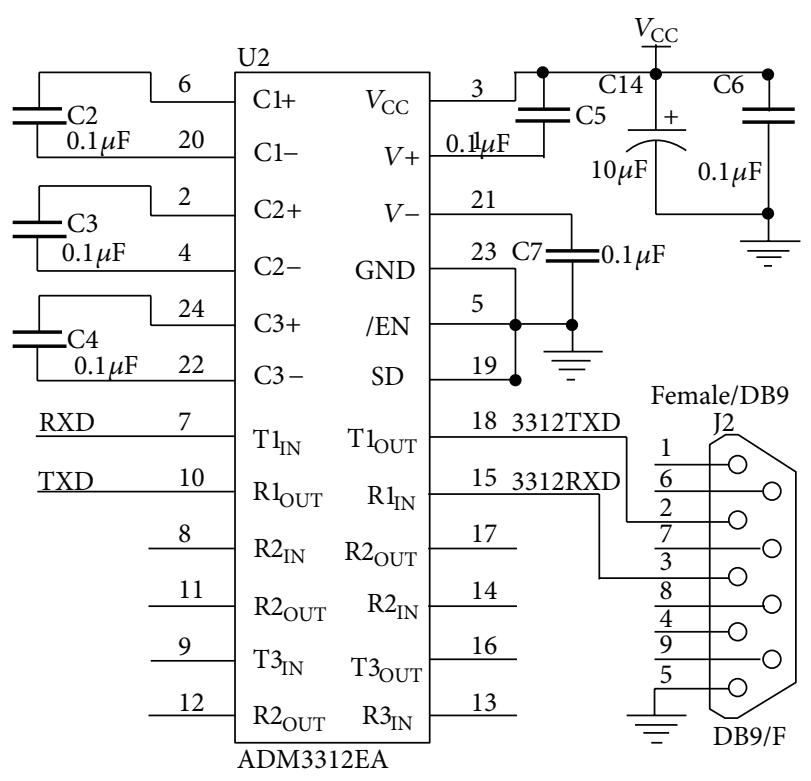

FIGURE 7: JN5139 chip and data transmission system circuit diagram.

is $3.3 \mathrm{~V}$. It is susceptible of outside surroundings as vehicle working environment is relatively poor, so it must be taken to voltage regulator measures. Selecting $\mu \mathrm{A} 7805 \mathrm{C}$ integrated voltage regulator chips and LM1117 chip, it can achieve two-stage voltage stabilization, and through three-way input, 


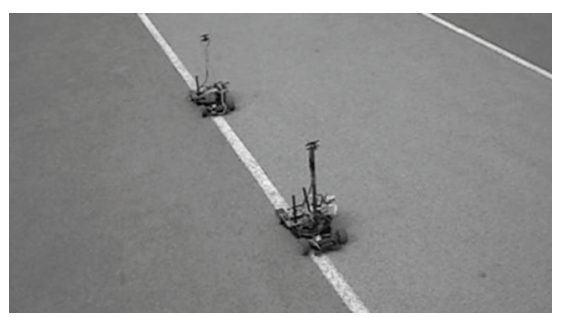

(a)

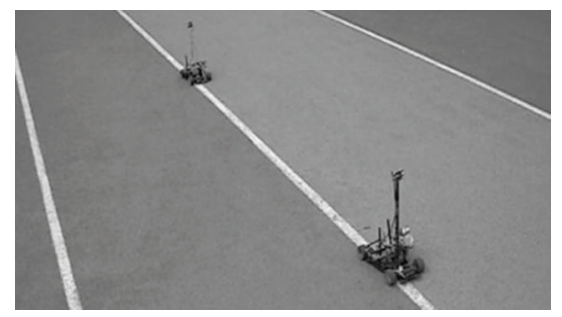

(b)

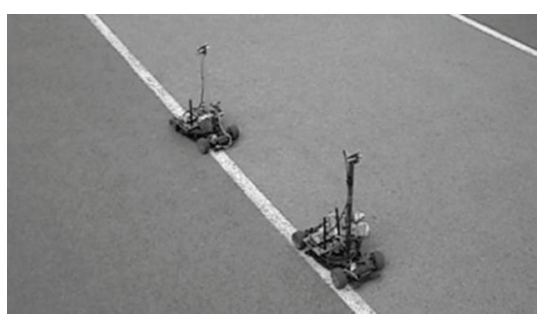

(c)

FIgURE 8: Experiment process.

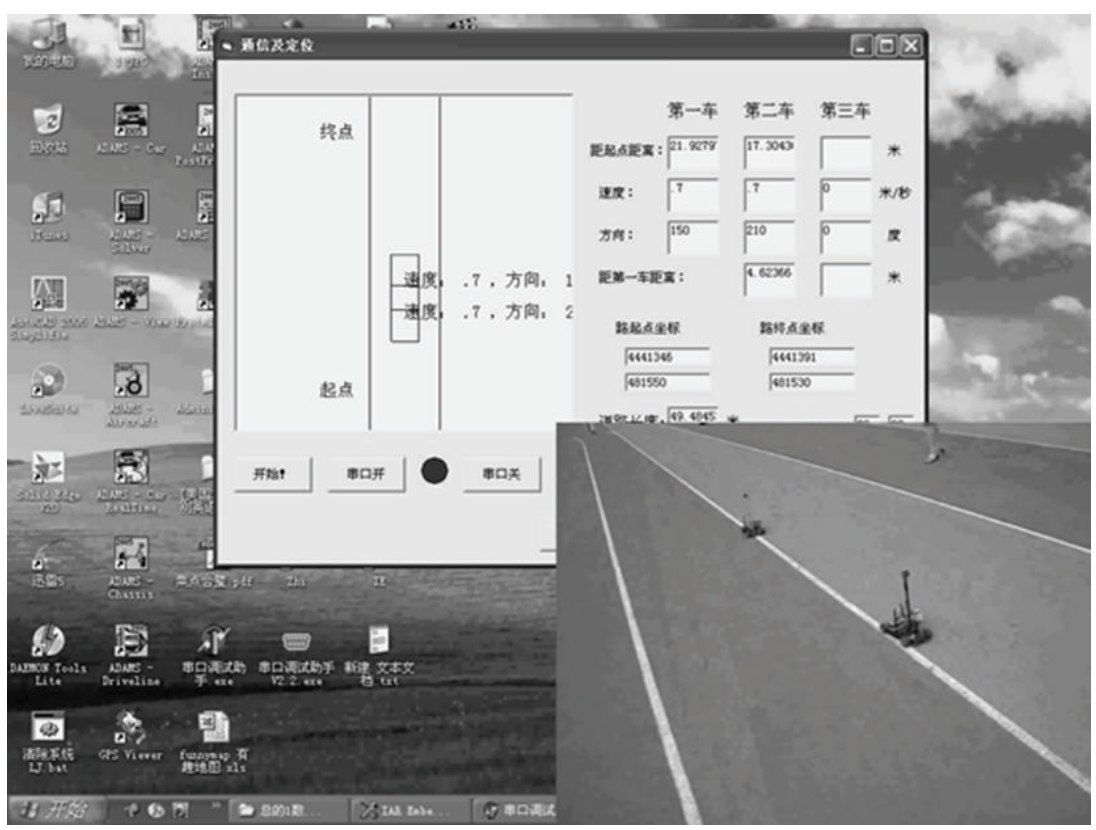

FigurE 9: Experiment data collecting process.

we can choose different input powers in different environments; the circuit is as shown in Figure 6.

3.4. Communication Module. This module is mainly used for wireless location and information transmission. Vehicle adjacent nodes analyze the receipt of the packet and extract the vehicle status information, and then they preprocess the extracted information and send it to the host computer program; relative relationship between multiple vehicles is displayed through the display module, including the position, velocity, and other information. Information collection and transmission are carried out by the JN5139 chip and its peripheral circuits; ADM3312 serial port chip is also used, as shown in Figure 7.

3.5. Vehicle Location Module. This module carries out vehicle location using wireless signals and acquires the vehicle location information by fusing GPS. This paper gets the approximate position of mobile node using triangle centroid method.

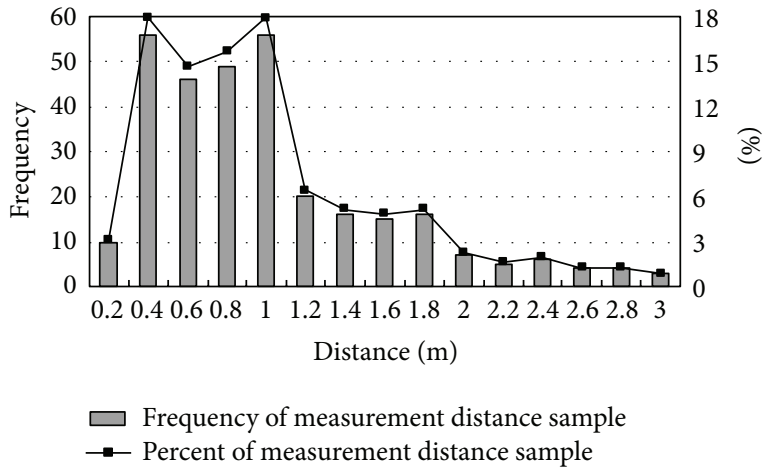

FIGURE 10: Two vehicles distance error distribution.

\section{Experimental Validation}

4.1. Experimental Schemes. Using the intervehicle communication experiment system, effectiveness of warning algorithm model will be verified. In the experimental process, the factor of driver type is not considered, taking $\gamma=0$. 


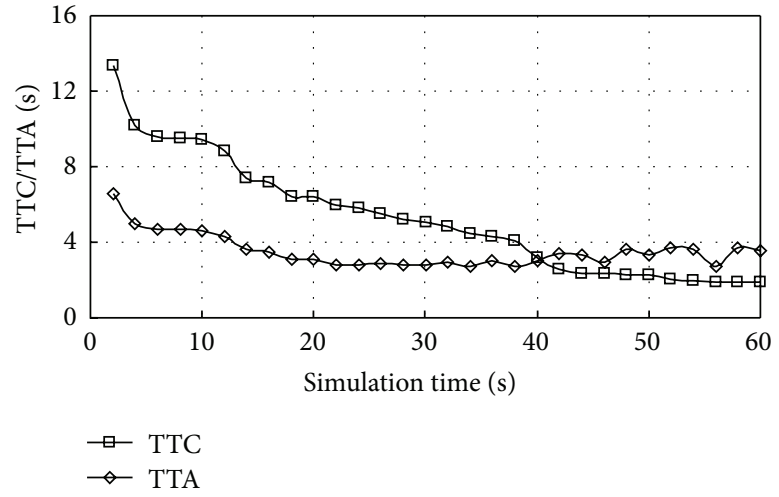

(a) Leading vehicle is stopping

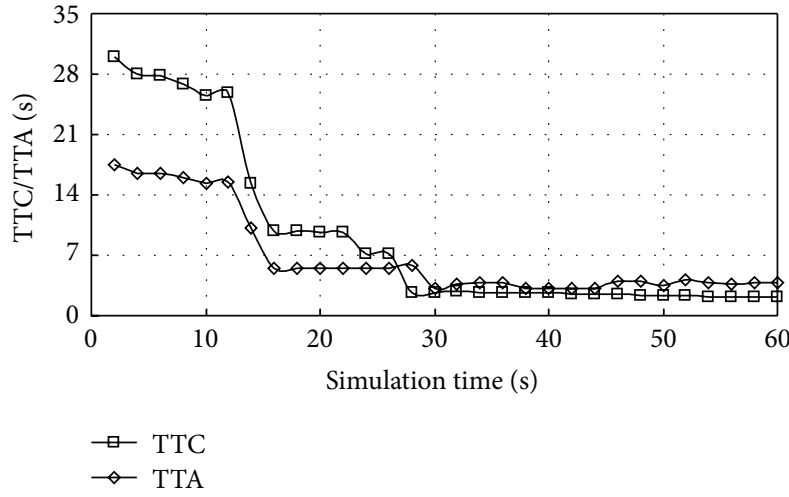

(b) Leading vehicle has a sudden deceleration until stopping

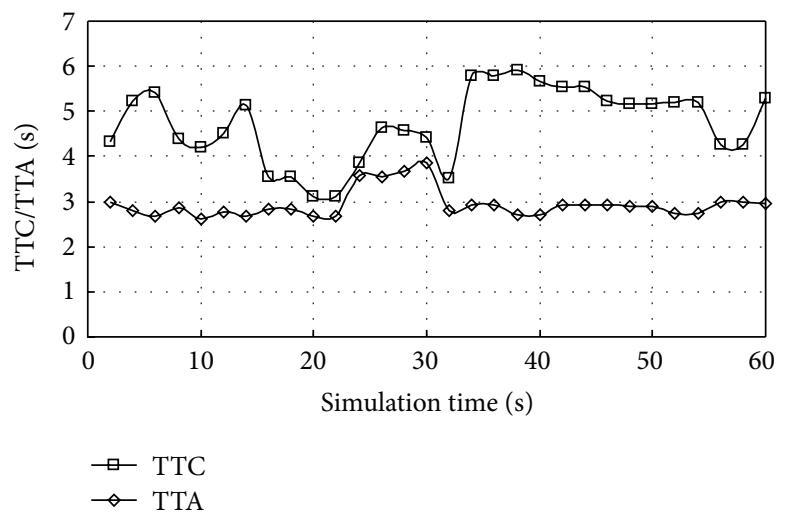

(c) Leading vehicle is normal running

FIGURE 11: TTC and TTA changing curves under three experimental programs.

The experiment schemes are as follows: (a) the leading vehicle stops and the following vehicle slowly approaches the leading vehicle $(\alpha=1)$, as shown in Figure 8(a); (b) the leading vehicle takes a sudden brake until stopping and the following vehicle also takes a sudden brake until stopping after reaction $(\alpha=0.8)$, as shown in Figure 8(b); (c) the two vehicles remain in normal vehicle running state ( $\alpha=0.5)$, as shown in Figure 8(c).

Using system software, one can realize real-time monitoring and acquire test sample data in the experimental process, as shown in Figure 9. According to the three experimental schemes, the corresponding experiments are conducted respectively, and the required sample data are obtained, which include the vehicle velocity, acceleration, displacement, signal strength, and so forth.

4.2. Warning Result Analysis. Using the experimental sample data, the distance error between two vehicles obtained by intervehicle communication is analyzed, as shown in Figure 10. It can be seen that the distance error is in the range of 0.4 1.0 meters mainly; however there are also some measurement data with relatively big error, which is occurred in the situation of leading vehicle's speed drastically changed.
The limitation of the algorithm and experiment system will be improved in future study.

According to the three proposed experimental schemes, different initial distance, velocity, and acceleration between vehicles are set, respectively. Real-time vehicle state information in experiment running process is collected, and TTC and TTA matrices are calculated based on the proposed algorithm. The experimental results are as shown in Figure 11.

The following can be seen from Figure 11: for the experimental scheme (a), TTC and TTA are in a slow decline process, and warning information is released in $42 \mathrm{~s}$; for the experimental scheme (b), leading vehicle has a sudden deceleration, TTC and TTA appear a fast drop course, and warning information is released in $26 \mathrm{~s}$; for the experimental scheme (c), two vehicles are in normal running state, relative distance has a relatively small change, TTC and TTA maintain a continuous process of change, and no warning information is released in the whole process.

4.3. Warning Performance Evaluation Indexes Analysis. In order to objectively evaluate model performance, two evaluation indexes are designed: proper warning rate and false warning rate. 
TABle 1: Performance evaluation index of warning model.

\begin{tabular}{|c|c|c|c|c|c|c|}
\hline & $R_{\mathrm{PW}} / \%$ & $R_{\mathrm{FW}} / \%$ & $R_{\mathrm{PW}} / \%$ & $R_{\mathrm{FW}} / \%$ & $R_{\mathrm{PW}} / \%$ & $R_{\mathrm{FW}} / \%$ \\
\hline & \multicolumn{2}{|c|}{ (a) The leading vehicle stops $(\alpha=1)$} & \multicolumn{2}{|c|}{$\begin{array}{l}\text { (b) The leading vehicle decelerates } \\
\text { suddenly }(\alpha=0.8)\end{array}$} & \multicolumn{2}{|c|}{$\begin{array}{l}\text { (c) The leading vehicle runs normally } \\
(\alpha=0.5)\end{array}$} \\
\hline \multirow[t]{2}{*}{ The paper } & 98.13 & 2.95 & 92.36 & 3.83 & 98.37 & 3.01 \\
\hline & \multicolumn{2}{|c|}{ Sunny conditions } & \multicolumn{2}{|c|}{ Rainy conditions } & \multicolumn{2}{|c|}{ Snow conditions } \\
\hline \multirow[t]{2}{*}{ Yi et al. [11] } & 98.2 & 1.8 & 98.2 & 1.6 & 98.6 & 1.8 \\
\hline & \multicolumn{2}{|c|}{ First grade } & \multicolumn{2}{|c|}{ Second grade } & \multicolumn{2}{|c|}{ Third grade } \\
\hline Wang et al. [12] & 98.13 & 2.9 & 98.73 & 3.8 & 92.63 & 3.0 \\
\hline
\end{tabular}

Proper warning rate $R_{\mathrm{PW}}$ is the ratio of the number of model identification warning and the total number of actual warning in a certain period. One has

$$
R_{\mathrm{PW}}=\frac{W_{P}}{W_{T}},
$$

where $W_{P}$ is the number of model identification warning in a certain period; $W_{T}$ is the total number of actual warning.

The false warning rate $R_{\mathrm{FW}}$ is the ratio of the false warning number and the total number of actual warning in a certain period, and the false warning includes the unidentified warning and false identified no-occurrence warning (excluding the false warning caused by hardware). One has

$$
R_{\mathrm{FW}}=\frac{W_{F}}{W_{T}},
$$

where $W_{F}$ is the number of false warnings in a certain period.

Table 1 presents the comparison results of three related models. The following can be seen from Table 1 .

(1) The model has a good warning performance for schemes (a) and (c) and has a relative bad warning performance for scheme (b). The main reason is that the intervehicle communication has a slow response on vehicle bake suddenly, and signal transmission delay has a relatively evident influence on distance between vehicles, which results in a relatively large error.

(2) The warning performance of the three warning modes is compared. The $R_{\mathrm{PW}}$ has an approximate same result, and the $R_{\mathrm{FW}}$ shows a difference. In comparison, the paper has a bigger $R_{\mathrm{FW}}$.

(3) The main reason is that the intervehicle communication signal is easily affected by external environment. The delay of data dissemination and data fluctuation causes the drop of experiment data in actual environment, and thus the warning result of revised paper shows a higher false warning rate $R_{\mathrm{FW}}$.

\section{Conclusion}

Integrating intervehicle communication to share vehicle state information under NLOS conditions, a collision warning algorithm is designed using the acceleration information and so forth, and then the algorithm mode is verified by the intervehicle communication experiment system. Experimental result has shown that the model can provide warning for the driver which can effectively improve vehicle safety. The contribution of the paper is the application of intervehicle communication on collision warning study and the design of the actual experiment system including the hardware and software systems. The paper will choose several typical warning models and compare the warning performance of the modes under conditions that meet the same standards. This is the next step to work on.

\section{Acknowledgments}

This work was financially supported by the National Natural Science Foundation (61104163, 71101008, and 71171014), the Fundamental Research Funds for the Central Universities (2012JBM066), and the National Basic Research Program of China (2012CB725403).

\section{References}

[1] http://www.mps.gov.cn/n16/n85753/n85870/index.html.

[2] H. Dong, W. Chen, M. Guo, and N. Chen, "A study on key technologies of vehicle active collision avoidance system based on VII technology," Automotive Engineering, vol. 32, no. 11, pp. 983-988, 2010.

[3] S. Biswas, R. Tatchikou, and F. Dion, "Vehicle-to-vehicle wireless communication protocols for enhancing highway traffic safety," IEEE Communications Magazine, vol. 44, no. 1, pp. 74$82,2006$.

[4] A. Tang and A. Yip, "Collision avoidance timing analysis of DSRC-based vehicles," Accident Analysis \& Prevention, vol. 42, no. 1, pp. 182-195, 2010.

[5] Y. Inoue, K. Ikeda, H. Mima et al., "A collision risk criterion based on imaginary brakes," in SICE Annual Conference, pp. 1358-1361, Taipei, Taiwan, August 2010.

[6] R. van der Horst and J. Hogema, "Time-to-collision and collision avoidance systems," in Proceedings of the 6th ICTCT Workshop on Safety Evaluation of Traffic Systems, pp. 109-112, Salzburg, Austria, 1993.

[7] R. Miller and Q. Huang, "An adaptive peer-to-peer collision warning system," in Proceedings of the 55th Vehicular Technology Conference, pp. 317-321, IEEE Press, Birmingham, Ala, USA, May 2002. 
[8] F. Gao, J. F. Wang, S. Y. Shi, and J. Wang, "Vehicle intelligent collision warning algorithm based on fuzzy neural networks," Journal of Jiangsu University, vol. 27, no. 3, pp. 211-215, 2006.

[9] P. Bucciol, J. L. Zechinelli-Martini, and G. Vargas-Solar, “Optimized transmission of loss tolerant information streams for real-time vehicle-to-vehicle communications," in Proceedings of the 10th Mexican International Conference on Computer Science (ENC '09), pp. 142-145, Puebla, Mexico, September 2009.

[10] D. Yu, Y.-H. Wu, X. He, and W.-L. Guo, "An car-following model based on dynamic desired time headway," Journal of Changsha University of Science and Technology, vol. 4, no. 4, pp. 25-28, 2007.

[11] F. J. Yi, Z. Han, and W. Deng, "An early-warning model for rearend accident occurring in highway tunnel group?" Journal of Tongji University, vol. 39, no. 11, pp. 1634-1640, 2011.

[12] J. Wang, F. Gao, and J. Wang, "Design of an innovative vehicle intelligent collision warning model," Journal of Beijing University of Aeronautics and Astronautics, vol. 33, no. 11, pp. 1325-1333, 2007. 


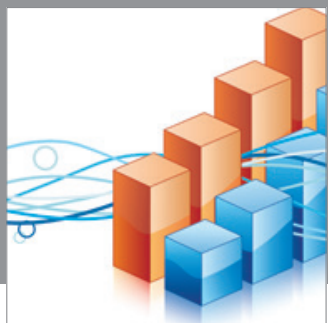

Advances in

Operations Research

mansans

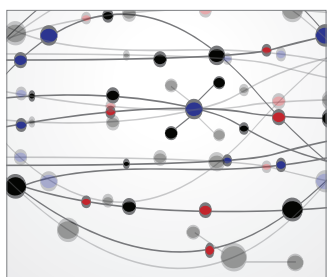

The Scientific World Journal
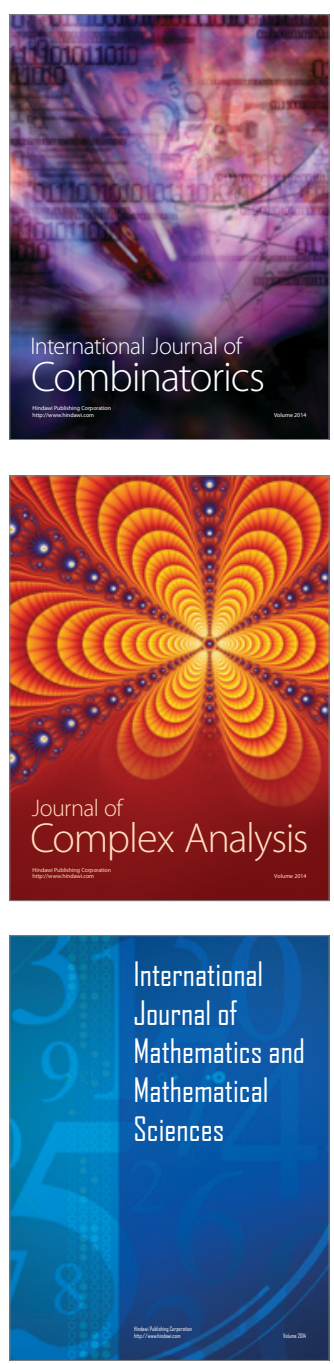
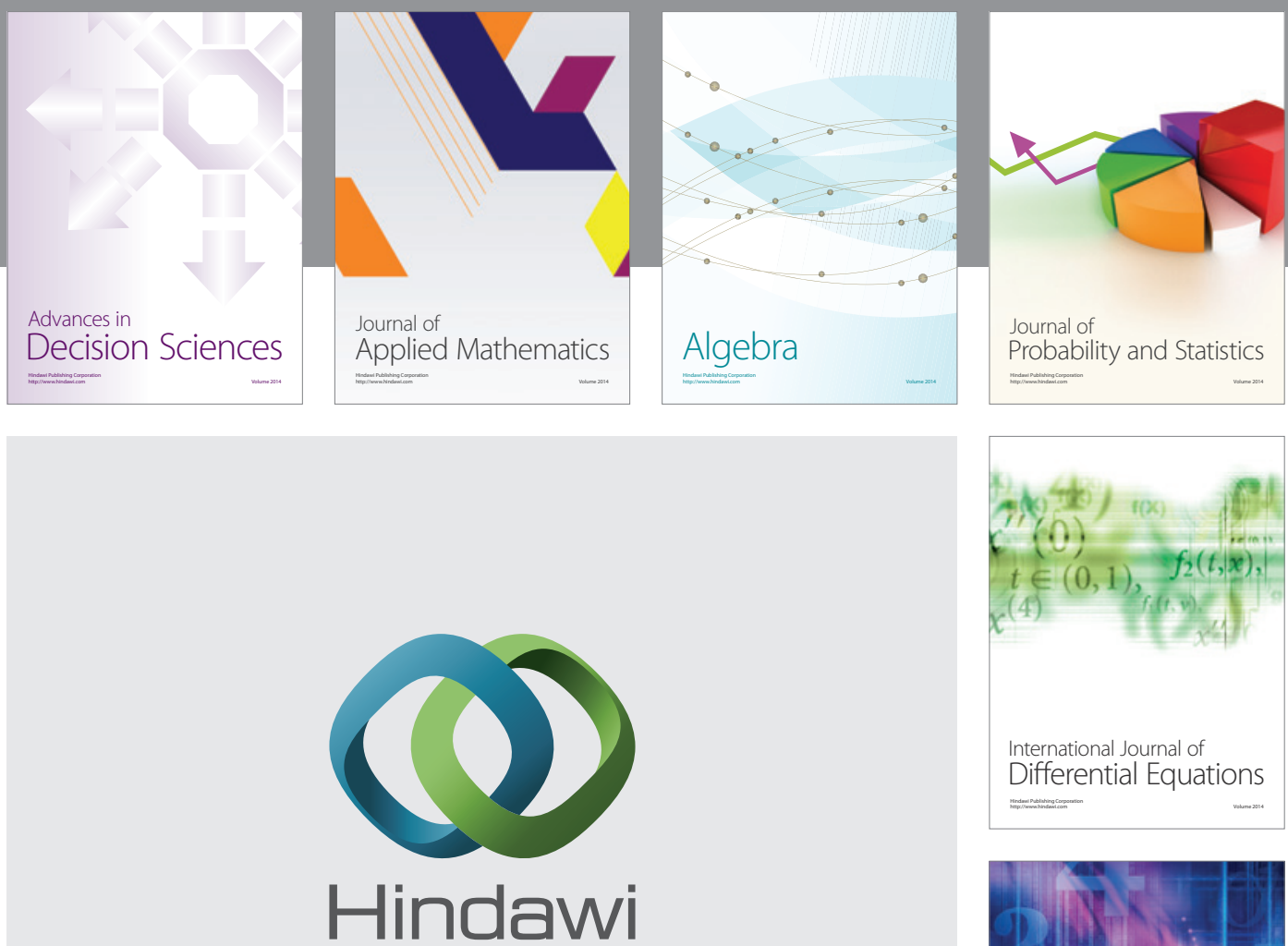

Submit your manuscripts at http://www.hindawi.com
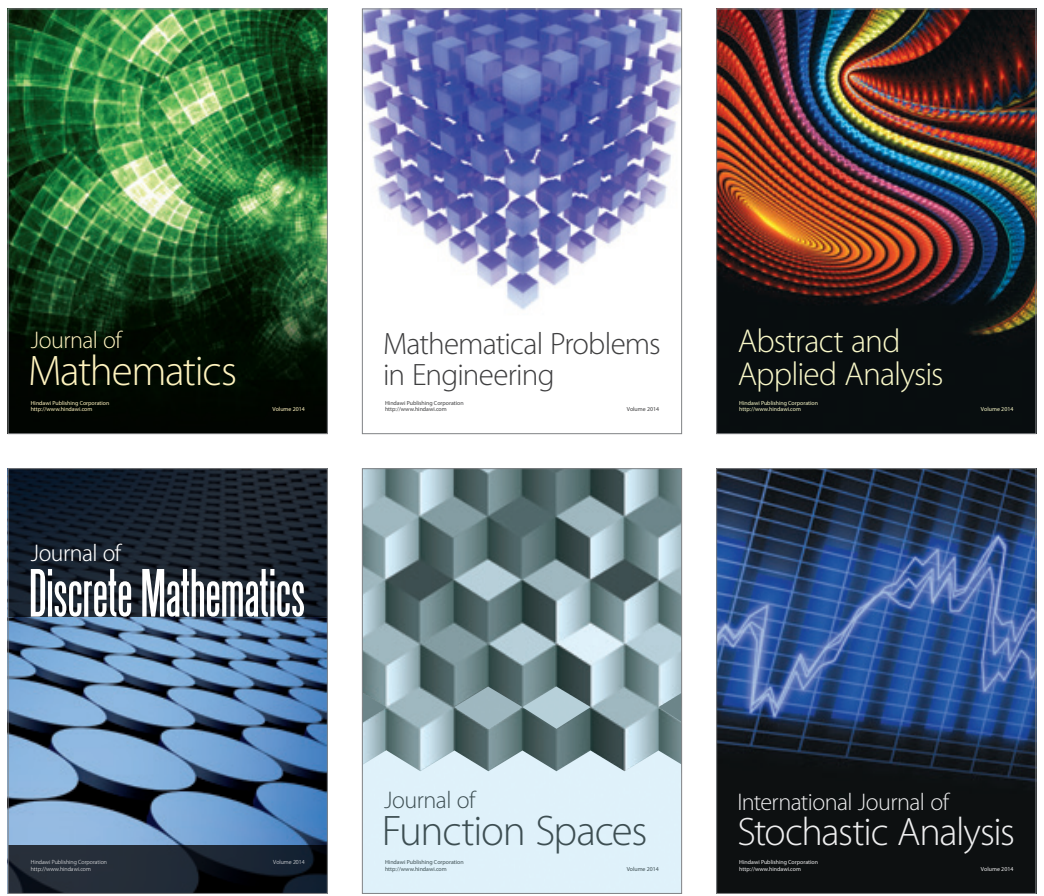

Journal of

Function Spaces

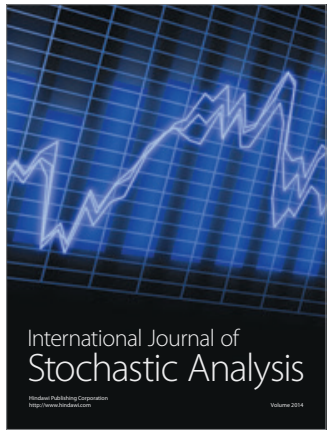

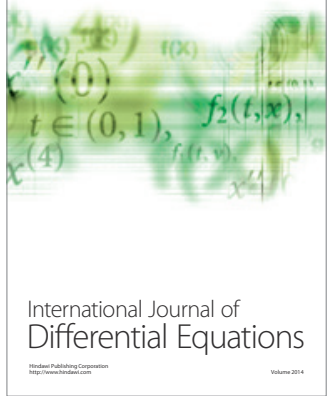
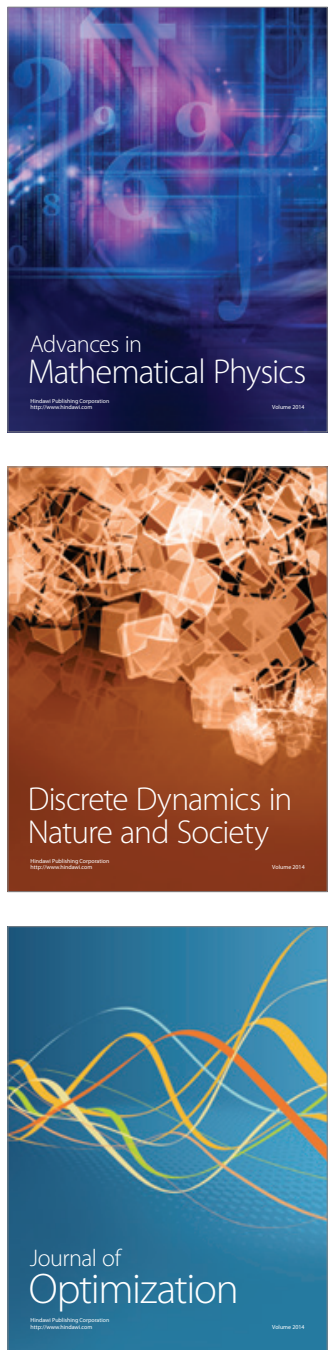